\title{
Curtius Rearrangement of Aromatic Carboxylic Acids to Access Protected Anilines and Aromatic Ureas
}

\section{Hélène Lebel* Olivier Leogane}

Département de chimie, Université de Montréal, Montréal, Québec, Canada, H3C 3J7

\author{
Supporting Information
}




\section{Table of Contents}

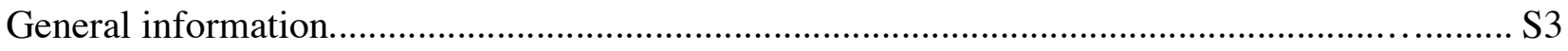

General procedure for the synthesis of aromatic Boc-carbamates ............................... 4

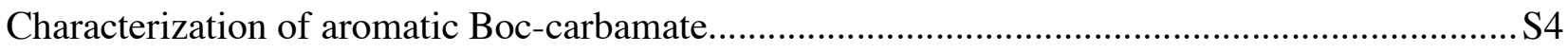

General procedure for the synthesis of aromatic Alloc-, CBz-, Troc-carbamates........................ S5

Characterization of aromatic Alloc-, CBz-, Troc-carbamates......................................................S5

General procedure for the synthesis of aromatic ureas..........................................S13

Characterization of aromatic ureas............................................................................ S13

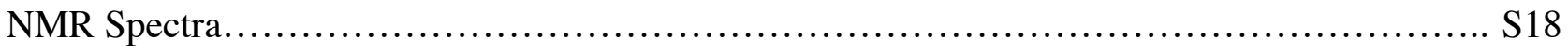


General: Unless otherwise noted, all non-aqueous reactions were performed under an oxygen-free atmosphere of argon with rigid exclusion of moisture from reagents and glassware. The solvents were dried using standard methods prior to use. $\mathrm{NaN}_{3}$ and sodium tert-butoxide are commercially available and were purchased from Aldrich. Sodium tert-butoxide was handled under inert atmosphere. 2-allylbenzoic acid was prepared from 2-bromobenzoic acid according to the literature. ${ }^{1}$ Other carboxylic acids are commercially available and were purified using standard methods prior to use. Analytical thin layer chromatography (TLC) was performed using EM Reagent $0.25 \mathrm{~mm}$ silica gel 60-F plates. Visualization of the developed chromatogram was performed by UV absorbance, aqueous cerium molybdate, ethanolic phosphomolybdic acid, or aqueous potassium permanganate. Flash chromatography was performed using EM Silica Gel 60 (230-400 mesh) with the indicated solvent system. Optical rotations were measured on a Perkin-Elmer 341 digital polarimeter at $589 \mathrm{~nm}$. Data are reported as follows: $[\alpha]_{\mathrm{D}}{ }^{\text {temp. }}$, concentration $(c \mathrm{~g} / 100 \mathrm{~mL})$, and solvent. ${ }^{1} \mathrm{H}$ NMR spectra were recorded in $\mathrm{CDCl}_{3}$, unless otherwise noted, on a Bruker AV-400, a Bruker ARX-400, a Bruker AMX300 or a Bruker AV-300 spectrometers (400, 400, 300 and $300 \mathrm{MHz}$ respectively). Chemical shifts are reported in ppm on the $\delta$ scale from an internal standard of residual chloroform (7.27 ppm). Data are reported as follows: chemical shift, multiplicity $(\mathrm{s}=$ singlet, $\mathrm{d}=$ doublet, $\mathrm{t}=$ triplet, $\mathrm{q}=$ quartet, $\mathrm{qn}=$ quintet, $\mathrm{m}=$ multiplet and $\mathrm{br}=$ broad), coupling constant in $\mathrm{Hz}$, integration. ${ }^{13} \mathrm{C} \mathrm{NMR}$ spectra were recorded in $\mathrm{CDCl}_{3}$, unless otherwise noted, on a Bruker AV-400, a Bruker ARX-400, a Bruker AMX300 or a Bruker AV-300 spectrometers $(100,100,75$ and $75 \mathrm{MHz}$ respectively) with complete proton decoupling. Chemical shifts are reported in ppm from the central peak of $\mathrm{CDCl}_{3}(77.0 \mathrm{ppm})$ on the $\delta$ scale. 2DCOSY experiments were realized for complex structures. Mass spectra were obtained on a LC-MSD TOF (ESI) Agilent Technologies high resolution from the Centre régional de spectrométrie de masse de l'Université de Montréal. Analytical gas chromatography with a mass spectroscopy (GCMS) was carried out on a Hewlett Packard 6890 series gas chromatograph equipped with a split mode capillary inJector and electron impact mass detector. Unless otherwise noted, inJector and detector temperatures were $250^{\circ} \mathrm{C}$ and the carrier gas was hydrogen $(2 \mathrm{~mL} / \mathrm{min})$ with a HP-5MS column. Data are reported as follows: column type, oven temperature, and retention time $\left(\mathrm{t}_{\mathrm{r}}\right)$.

1 a) Inoue, A.; Kitawaga,, K.; Shinorubo, H.; Oshima, K. J. Org. Chem. 2001, 66, 4333-4339. b) Bauer, M.; Maier, M. E. Org. Lett. 2002, 4, 2205-2208. 


\section{General procedure for the synthesis of aromatic Boc-carbamates}

To a solution of sodium azide $(0.097 \mathrm{~g}, 1.50 \mathrm{mmol})$, and carboxylic acid $(1.00 \mathrm{mmol})$ in DME (10.0 $\mathrm{mL})$ at $25^{\circ} \mathrm{C}$, was added di-tert-butyldicarbonate $(250 \mu \mathrm{L}, 1.10 \mathrm{mmol})$. Then the resulting mixture was stirred at $75^{\circ} \mathrm{C}$. When the reaction is completed by GC analysis, a $10 \%$ solution of $\mathrm{NaNO}_{2}(20$ $\mathrm{mL})$ was added. The mixture was then diluted with chloroform $(20 \mathrm{ml})$, and the resulting mixture was stirred during $20 \mathrm{~min}$ at $25^{\circ} \mathrm{C}$. The aqueous layer was acidified with a $10 \%$ solution of $\mathrm{HCl}$ to $\mathrm{pH}=4$ and then extracted with chloroform $(3 \times 30 \mathrm{~mL})$ The combined organic layers were washed with saturated $\mathrm{NH}_{4} \mathrm{Cl}(2 \times 20 \mathrm{~mL})$, and brine $(20 \mathrm{~mL})$. The organic layer was dried over $\mathrm{Na}_{2} \mathrm{SO}_{4}$ and the solvent was removed under reduced pressure and the crude carbamate was purified by flash chromatography on silica gel with a pre-absorption on silica

NB. Although we have conducted this procedure on a $10 \mathrm{mmol}$ scale without any problem, extreme care should be taken when carried out this reaction, as potentially explosive azide-containing intermediates could be generated under these reaction conditions.

\section{Characterization of aromatic Boc-carbamate}

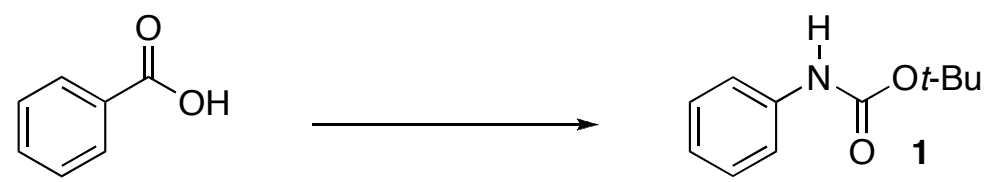

tert-Butyl phenylcarbamate (1). The title compound was prepared from benzoic acid (122 mg, 1.00 $\mathrm{mmol})$ according to the general procedure. The desired carbamate $\mathbf{1}(152 \mathrm{mg}, 79 \%)$ was obtained as a white solid after flash chromatography $\left(10 \%\right.$ EtOAc/hexanes). $\mathrm{R}_{f} 0.62$ (20\% EtOAc/hexanes); mp $130{ }^{\circ} \mathrm{C} ;{ }^{1} \mathrm{H}$ NMR $\left(400 \mathrm{MHz}, \mathrm{CDCl}_{3}\right) \delta$ 7.39-7.37 (m, 2H), 7.33-7.29 (m, 2H), 7.07-7.04 (m, 1H), 6.50 (s (br), 1H), 1.54 (s, 9H); ${ }^{13} \mathrm{C} \mathrm{NMR}\left(100 \mathrm{MHz}, \mathrm{CDCl}_{3}\right) \delta$ 152.7, 138.3, 128.9, 122.9, 118.5, 80.4, 28.3; IR 3301, 3042, 2983, 2933, 1684, 1597, 1527, 1439, 1242, 1147, 1054, 743, $691 \mathrm{~cm}^{-1}$; HMRS (ESI) calcd for $\mathrm{C}_{11} \mathrm{H}_{15} \mathrm{NO}_{2} \mathrm{Na}[\mathrm{M}+\mathrm{Na}]^{+}:$216.0995. Found: 216.0990 .

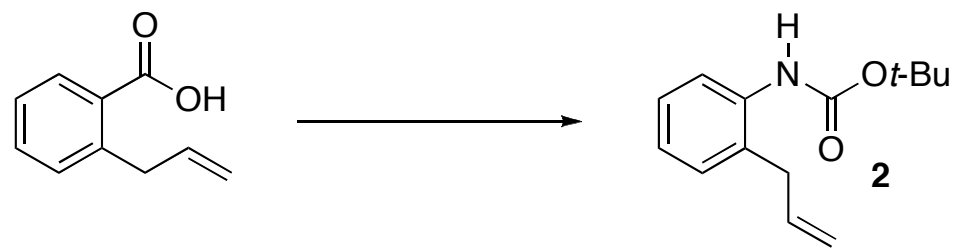

tert-Butyl 2-allylphenylcarbamate (2). The title compound was prepared from 2-allylbenzoic acid (162 $\mathrm{mg}, 1.00 \mathrm{mmol})$ according to the general procedure. The desired carbamate $2(177 \mathrm{mg}, 76 \%)$ was obtained as a colorless oil after flash chromatography (10\% EtOAc/hexanes). $\mathrm{R}_{f} \quad 0.71$ (20\% EtOAc/hexanes); ${ }^{1} \mathrm{H}$ NMR $\left(400 \mathrm{MHz}, \mathrm{CDCl}_{3}\right) \delta 7.81(\mathrm{~d}, J=7 \mathrm{~Hz}, 1 \mathrm{H}), 7.26(\mathrm{t}, J=8 \mathrm{~Hz}, 1 \mathrm{H}), 7.17(\mathrm{~d}, J$ $=7 \mathrm{~Hz}, 1 \mathrm{H}), 7.07(\mathrm{t}, J=7 \mathrm{~Hz}, 1 \mathrm{H}), 6.48(\mathrm{~s}(\mathrm{br}), 1 \mathrm{H}), 5.99(\mathrm{ddt}, J=17,10,6 \mathrm{~Hz}, 1 \mathrm{H}), 5.20-5.17(\mathrm{~m}, 1 \mathrm{H})$, 5.11-5.07 (m, 1H), $3.39(\mathrm{~d}, J=6 \mathrm{~Hz}, 2 \mathrm{H}), 1.54(\mathrm{~s}, 9 \mathrm{H}) ;{ }^{13} \mathrm{C}$ NMR $\left(100 \mathrm{MHz}, \mathrm{CDCl}_{3}\right) \delta 153.2,136.4$, 135.8, 130.0, 127.3, 123.9, 121.9, 116.5, 80.3, 36.5, 28.3; IR 3356, 2968, 2918, 1691, 1520, 1430, 1232, 1153, 1075, 1010, 842, $687 \mathrm{~cm}^{-1}$; HMRS (ESI) calcd for $\mathrm{C}_{14} \mathrm{H}_{19} \mathrm{NO}_{2}[\mathrm{M}+\mathrm{Na}]^{+}:$256.1308. Found: 256.1302 . 
<smiles>CCCOC(=O)Nc1cc(C)cc(C)c1</smiles>

tert-Butyl 3,5-dimethylphenylcarbamate (3). The title compound was prepared from 3,5-dimethylbenzoic acid (150 mg, $1.00 \mathrm{mmol}$ ) according to the general procedure. The desired carbamate 3 (163 $\mathrm{mg}, 74 \%)$ was obtained as a white solid after flash chromatography (10\% EtOAc/hexanes). $\mathbf{R}_{f} 0.69$ (20\% EtOAc/hexanes); mp $73{ }^{\circ} \mathrm{C} ;{ }^{1} \mathrm{H}$ NMR (400 MHz, $\mathrm{CDCl}_{3}$ ) $\delta 7.03$ (s (br), 2H), 6.71 (s (br), 1H),

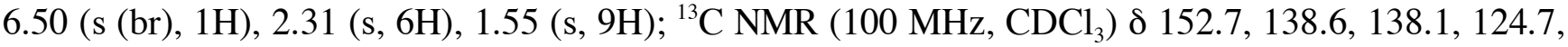
116.2, 80.2, 28.3, 21.3; IR 3356, 2968, 2918, 1691, 1520, 1430, 1232, 1153, 1075, 1010, 842, $687 \mathrm{~cm}^{-1}$; HMRS (ESI) calcd for $\mathrm{C}_{13} \mathrm{H}_{19} \mathrm{NO}_{2}[\mathrm{M}+\mathrm{Na}]^{+}:$244.1308. Found: 244.1298.

\section{General procedure for the synthesis of aromatic Alloc-, CBz-, Troc-carbamates}

To a solution of sodium azide $(0.110 \mathrm{~g}, 1.70 \mathrm{mmol})$, sodium tert-butoxide $(14.4 \mathrm{mg}, 0.150 \mathrm{mmol})$, and carboxylic acid $(1.00 \mathrm{mmol})$ in DME $(10.0 \mathrm{~mL})$ at $25^{\circ} \mathrm{C}$, was added the chloroformate reagent $(1.10$ mmol) (Allylchloroformate: $120 \mu \mathrm{L}$, benzylchloroformate: $150 \mu \mathrm{L}$, or 2,2,2 trichloroethylchloroformate: $112 \mu \mathrm{L})$. Then the resulting mixture was stirred at $75^{\circ} \mathrm{C}\left(85^{\circ} \mathrm{C}\right.$ for Troc derivatives). When the reaction is completed by GC analysis, the mixture was diluted with dichloromethane and a preabsorption on silica was made. The crude carbamate was then purified by flash chromatography on silica gel.

\section{Characterization of aromatic Alloc-, CBz-, Troc-carbamates}<smiles>O=C(O)c1ccccc1</smiles><smiles>C=CCOC(=O)Nc1ccccc1</smiles>

Allyl phenylcarbamate (4). The title compound was prepared from benzoic acid (122 $\mathrm{mg}, 1.00 \mathrm{mmol})$ according to the general procedure. The desired carbamate $4(101 \mathrm{mg}, 57 \%)$ was obtained as a white solid after flash chromatography $\left(10 \%\right.$ EtOAc/hexanes). $\mathrm{R}_{f} 0.45$ (20\% EtOAc/hexanes); mp $48{ }^{\circ} \mathrm{C} ;{ }^{1} \mathrm{H}$ NMR (400 MHz, $\left.\mathrm{CDCl}_{3}\right) \delta$ 7.40-7.38 (m, 2H), 7.33-7.29 (m, 2H), 7.07 (t, $\left.J=7 \mathrm{~Hz}, 1 \mathrm{H}\right), 6.70$ (s (br), $1 \mathrm{H}), 5.97(\mathrm{ddt}, \quad J=17,10,6 \mathrm{~Hz}, 1 \mathrm{H}), 5.39-5.34(\mathrm{~m}, 1 \mathrm{H}), 5.28-5.25(\mathrm{~m}, 1 \mathrm{H}), 4.68-4.66(\mathrm{~m}, 2 \mathrm{H}) ;{ }^{13} \mathrm{C}$ NMR $\left(100 \mathrm{MHz}, \mathrm{CDCl}_{3}\right) \delta 153.2,137.7,132.4,129.0,123.4,118.7,118.1,65.8$; IR 3302, 3061, 2955, 1701, 1596, 1533, 1442, 1222, 1054, 936, 743, $692 \mathrm{~cm}^{-1}$; HMRS (ESI) calcd for $\mathrm{C}_{10} \mathrm{H}_{12} \mathrm{NO}_{2}[\mathrm{M}+\mathrm{H}]^{+}$: 178.0863. Found: 178.0866.<smiles>O=C(O)c1ccccc1</smiles>

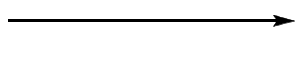<smiles>O=C(Nc1ccccc1)OCc1ccccc1</smiles>

Benzyl phenylcarbamate (5). The title compound was prepared from benzoic acid (122 mg, 1.00 mmol) according to the general procedure. The desired carbamate 5 (145 $\mathrm{mg}, 64 \%)$ was obtained after flash chromatography (15\% EtOAc/hexanes), as a white solid. $\mathrm{R}_{f} 0.49$ (20\% EtOAc/hexanes); mp $38{ }^{\circ} \mathrm{C} ;{ }^{1} \mathrm{H}$ NMR (400 MHz, $\left.\mathrm{CDCl}_{3}\right)$ d 7.42-7.29 (m, 9H), 7.10-7.05 (m, 1H), 6.70 (s (br), 1H), 5.21 (s, $2 \mathrm{H}) ;{ }^{13} \mathrm{C} \mathrm{NMR}\left(100 \mathrm{MHz}, \mathrm{CDCl}_{3}\right) \delta 153.2,137.7,132.4,129.05,128.96$ (2C), 123.4, 118.6, 118.1, 65.8; IR 3267, 3135, 3076, 2953, 2894, 1686, 1598, 1542, 1443, 1315, 1236, 1223, 1055, 845,735, 693 $\mathrm{cm}^{-1}$; HMRS (ESI) calcd for $\mathrm{C}_{14} \mathrm{H}_{14} \mathrm{NO}_{2}[\mathrm{M}+\mathrm{H}]^{+}: 228.1020$. Found: 228.1019. 


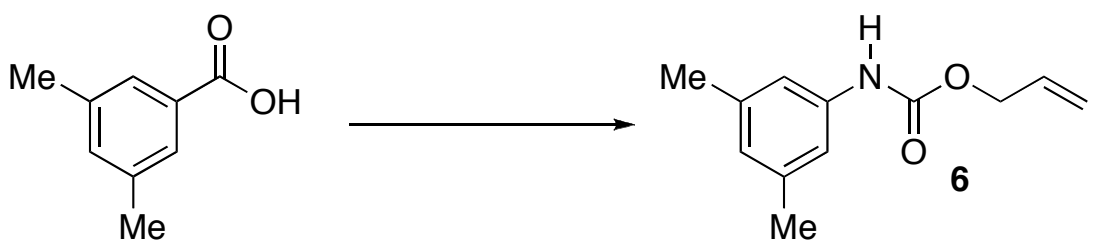

Allyl 3,5-dimethylphenylcarbamate (6). The title compound was prepared from 3,5-dimethylbenzoic acid (150 mg, $1.00 \mathrm{mmol}$ ) according to the general procedure. The desired carbamate 6 (135 $\mathrm{mg}, 66 \%)$ was obtained as a white solid after flash chromatography (10\% EtOAc/hexanes). $\mathrm{R}_{f} 0.50$ (20\% EtOAc/hexanes); mp $37{ }^{\circ} \mathrm{C}$; ${ }^{1} \mathrm{H}$ NMR (400 MHz, CDCl ${ }_{3}$ ) 8.04 (s (br), 2H), 6.80 (s (br), 1H), 6.72 (s (br), 1H), 6.03-5.93 (m, 1H), 5.39-5.35 (m, 1H), 5.28-5.25 (m, 1H), 4.68-4.66 (m, 2H), $2.29(\mathrm{~s}, 6 \mathrm{H})$; ${ }^{13} \mathrm{C}$ NMR $\left(100 \mathrm{MHz}, \mathrm{CDCl}_{3}\right) \delta$ 153.2, 138.6, 137.5, 132.4, 125.1, 117.9, 116.4, 65.6, 21.2; IR 3316, 2919, 1704, 1614, 1548, 1450, 1218, 1084, 931, 841, $769 \mathrm{~cm}^{-1}$; HMRS (ESI) calcd for $\mathrm{C}_{12} \mathrm{H}_{16} \mathrm{NO}_{2}$ $[\mathrm{M}+\mathrm{H}]^{+}:$206.1176. Found: 206.1168.<smiles>Cc1cc(C)cc(C(=O)O)c1</smiles><smiles>Cc1cc(C)cc(NC(=O)OCc2ccccc2)c1</smiles>

Benzyl 3,5-dimethylphenylcarbamate (7). The title compound was prepared from 3,5-dimethylbenzoic acid $(150 \mathrm{mg}, 1.00 \mathrm{mmol})$ according to the general procedure. The desired carbamate 7 (148 $\mathrm{mg}, 58 \%$ ) was obtained as a white solid after flash chromatography (20\% EtOAc/hexanes). $\mathrm{R}_{f} 0.49$ (20\% EtOAc/hexanes); mp $43{ }^{\circ} \mathrm{C} ;{ }^{1} \mathrm{H}$ NMR (400 MHz, $\mathrm{CDCl}_{3}$ ) $\delta$ 7.42-7.33 (m, 5H), 7.02 (s (br), 2H), 6.72 (s (br), 1H), 6.60 (s (br), 1H), 5.19 (s, 2H), 2.29 (s, 6H); $\left.{ }^{13} \mathrm{C} \mathrm{NMR} \mathrm{(100} \mathrm{MHz,} \mathrm{CDCl}{ }_{3}\right) \delta 153.3$, 138.7, 137.5, 136.1, 128.5, 128.2, 125.2, 116.4, 66.8, 21.3; IR 3289, 3034, 2917, 1694, 1531, 1453, $1269,1215,1078,838,739,685 \mathrm{~cm}^{-1}$; HMRS (ESI) calcd for $\mathrm{C}_{16} \mathrm{H}_{18} \mathrm{NO}_{2}[\mathrm{M}+\mathrm{H}]^{+}:$: 256.1333. Found: 256.1332 .<smiles>O=C(O)c1ccc2ccccc2c1</smiles><smiles>C=CCOC(=O)Nc1ccc2ccccc2c1</smiles>

Allyl naphthalen-3-ylcarbamate (8). The title compound was prepared from 2-naphtanoic acid (172 $\mathrm{mg}, 1.00 \mathrm{mmol})$ according to the general procedure. The desired carbamate $8(175 \mathrm{mg}, 77 \%)$ was obtained as a pale brown solid after flash chromatography (20\% EtOAc/hexanes). $\mathrm{R}_{f} 0.31$ (20\% EtOAc/hexanes); mp $98{ }^{\circ} \mathrm{C} ;{ }^{1} \mathrm{H} \mathrm{NMR}\left(400 \mathrm{MHz}, \mathrm{CDCl}_{3}\right) \delta 7.90-7.88(\mathrm{~m}, 3 \mathrm{H}), 7.69(\mathrm{~d}, J=8 \mathrm{~Hz}, 1 \mathrm{H})$, 7.54-7.47 (m, 3H), $7.13(\mathrm{~s}(\mathrm{br}), 1 \mathrm{H}), 6.09-5.99(\mathrm{~m}, 1 \mathrm{H}), 5.42(\mathrm{~d}, J=17 \mathrm{~Hz}, 1 \mathrm{H}), 5.31(\mathrm{~d}, J=10 \mathrm{~Hz}$, $1 \mathrm{H}), 4.76(\mathrm{~d}, J=6 \mathrm{~Hz}, 2 \mathrm{H}) ;{ }^{13} \mathrm{C}$ NMR $\left(100 \mathrm{MHz}, \mathrm{CDCl}_{3}\right) \delta 154.1,133.9,132.4,132.3,128.6,126.7$, 126.1, 125.9, 125.7, 125.0, 120.4, 119.1, 118.2, 66.0 ; IR 3265, 3050, 2940, 1693, 1525, 1501, 1253, 1230, 1103, 990, 785, $764 \mathrm{~cm}^{-1}$; HMRS (ESI) calcd for $\mathrm{C}_{14} \mathrm{H}_{14} \mathrm{NO}_{2}[\mathrm{M}+\mathrm{H}]^{+}:$228.1024. Found: 228.1019.<smiles>O=C(O)c1ccc2ccccc2c1</smiles><smiles>O=C(Nc1ccc2ccccc2c1)OCc1ccccc1</smiles>

Benzyl naphthalen-3-ylcarbamate (9). The title compound was prepared from 2-naphtanoic acid (172 $\mathrm{mg}, 1.00 \mathrm{mmol}$ ) according to the general procedure. The desired carbamate $9(152 \mathrm{mg}, 55 \%)$ was 
obtained after flash chromatography (20\% EtOAc/hexanes), as a pale brown solid. $\mathrm{R}_{f} 0.30$ (20\% EtOAc/hexanes); mp $117{ }^{\circ} \mathrm{C} ;{ }^{1} \mathrm{H}$ NMR (400 MHz, $\left.\mathrm{CDCl}_{3}\right) \delta 7.91-7.89(\mathrm{~m}, 3 \mathrm{H}), 7.71(\mathrm{~d}, \mathrm{~J}=8 \mathrm{~Hz}, 1 \mathrm{H})$,

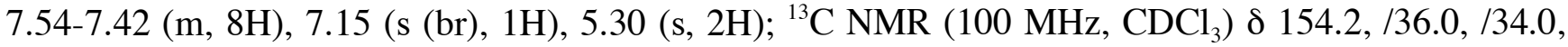
132.3, 128.6, 128.5, 128.32, 128.29, 126.6, 126.1, 126.0, 125.7, 125.0, 120.4, 118.9, 67.2; IR 3277, 3031, 2950, 1688, 1534, 1502, 1235, 1068, 766, $694 \mathrm{~cm}^{-1}$; HMRS (ESI) calcd for $\mathrm{C}_{18} \mathrm{H}_{16} \mathrm{NO}_{2}[\mathrm{M}+\mathrm{H}]^{+}$: 278.1188. Found: 278.1175.

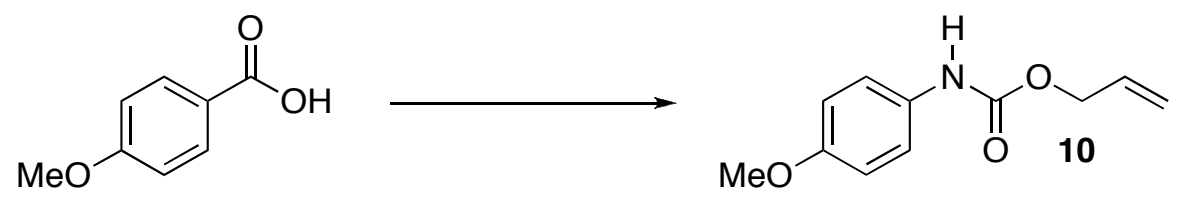

Allyl 4-methoxyphenylcarbamate (10). The title compound was prepared from 4-methoxybenzoic acid (152 mg, $1.00 \mathrm{mmol}$ ) according to the general procedure. The desired carbamate 10 (93 $\mathrm{mg}, 45 \%)$ was obtained as a pale yellow oil after flash chromatography $\left(20 \%\right.$ EtOAc/hexanes). $\mathrm{R}_{f} 0.30(20 \%$ EtOAc/hexanes); ${ }^{1} \mathrm{H} \mathrm{NMR}\left(400 \mathrm{MHz}, \mathrm{CDCl}_{3}\right.$ ) $\delta 7.29$ (s (br), 2H), 6.83 (d, J = 9Hz, 2H), 6.80 (s (br), $1 \mathrm{H}), 5.94(\mathrm{ddt}, J=17,10,6 \mathrm{~Hz}, 1 \mathrm{H}), 5.35(\mathrm{~d}, J=17 \mathrm{~Hz}, 1 \mathrm{H}), 5.24(\mathrm{~d}, J=10 \mathrm{~Hz}, 1 \mathrm{H}), 4.64(\mathrm{~d}, J=6$ $\mathrm{Hz}, 2 \mathrm{H}), 3.77$ (s, 3H); ${ }^{13} \mathrm{C} \mathrm{NMR}\left(100 \mathrm{MHz}, \mathrm{CDCl}_{3}\right) \delta 155.9,153.6,132.5,130.8,120.6,118.0,114.1$, 65.7, 55.4; IR 3310, 2933, 1703, 1514, 1414, 1221, 1033, $828 \mathrm{~cm}^{-1}$; HMRS (ESI) calcd for $\mathrm{C}_{11} \mathrm{H}_{14} \mathrm{NO}_{3}$ $[\mathrm{M}+\mathrm{H}]^{+}:$208.0960. Found: 208.0968.<smiles>CC(C)(C)c1ccc(C(=O)O)cc1</smiles><smiles>C=CCOC(=O)Nc1ccc(C(C)(C)C)cc1</smiles>

Allyl 4-tert-butylphenylcarbamate (11). The title compound was prepared from 4-tert-butyl benzoic acid (178 $\mathrm{mg}, 1.00 \mathrm{mmol}$ ) according to the general procedure. The desired carbamate 11 (130 $\mathrm{mg}, 56$ $\%)$ was obtained as a white solid after flash chromatography (10\% EtOAc/hexanes). $\mathrm{R}_{f} 0.52(20 \%$ EtOAc/hexanes); mp $72{ }^{\circ} \mathrm{C} ;{ }^{1} \mathrm{H}$ NMR (400 MHz, CDCl ${ }_{3}$ ) 7.33 (s (br), 4H), 6.81 (s (br), 1H), 5.98 (ddt, $J=17,10,6 \mathrm{~Hz}, 1 \mathrm{H}), 5.36(\mathrm{~d}, J=17 \mathrm{~Hz}, 1 \mathrm{H}), 5.26(\mathrm{~d}, J=10 \mathrm{~Hz}, 1 \mathrm{H}), 4.67(\mathrm{~d}, J=6 \mathrm{~Hz}, 2 \mathrm{H})$, 1.31 (s, 9H); ${ }^{13} \mathrm{C}$ NMR $\left(100 \mathrm{MHz}, \mathrm{CDCl}_{3}\right) \delta 153.4,146.3,135.1,132.5,125.8,118.5,118.0,65.7,34.2$, 31.3; IR 3326, 2964, 2868, 1698, 1529, 1407, 1226, 1054, 992, 836, $768 \mathrm{~cm}^{-1}$; HMRS (ESI) calcd for $\mathrm{C}_{14} \mathrm{H}_{20} \mathrm{NO}_{2}[\mathrm{M}+\mathrm{H}]^{+}:$234.1495. Found: 234.1488.<smiles>COc1ccc(C(=O)O)cc1</smiles>

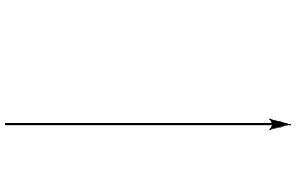<smiles>COc1ccc(NC(=O)OCc2ccccc2)cc1</smiles>

Benzyl 4-methoxyphenylcarbamate (12). The title compound was prepared from 4-methoxybenzoic acid $(152 \mathrm{mg}, 1.00 \mathrm{mmol})$ according to the general procedure. The desired carbamate $12(159 \mathrm{mg}$, $62 \%$ ) was obtained after flash chromatography (20\% EtOAc/hexanes), as a white solid. $\mathrm{R}_{f} 0.20(20 \%$ EtOAc/hexanes); mp $88{ }^{\circ} \mathrm{C} ;{ }^{1} \mathrm{H}$ NMR $\left(400 \mathrm{MHz}, \mathrm{CDCl}_{3}\right) \delta 7.41-7.28(\mathrm{~m}, 8 \mathrm{H}), 6.87(\mathrm{~d}, J=9 \mathrm{~Hz}, 2 \mathrm{H})$, $5.21(\mathrm{~s}, 2 \mathrm{H}), 3.80(\mathrm{~s}, 3 \mathrm{H}) ;{ }^{13} \mathrm{C} \mathrm{NMR}\left(100 \mathrm{MHz}, \mathrm{CDCl}_{3}\right) \delta 155.8,153.7,136.1,130.8,128.5,128.1$ (2C), 120.6, 114.1, 66.8, 55.4; IR 3303, 3041, 2969, 2843, 1699, 1526, 1511, 1414, 1234, 1187, 1063, 1027 , $823,741 \mathrm{~cm}^{-1}$; HMRS (ESI) calcd for $\mathrm{C}_{15} \mathrm{H}_{16} \mathrm{NO}_{3}[\mathrm{M}+\mathrm{H}]^{+}:$258.1125. Found: 258.1131 . 


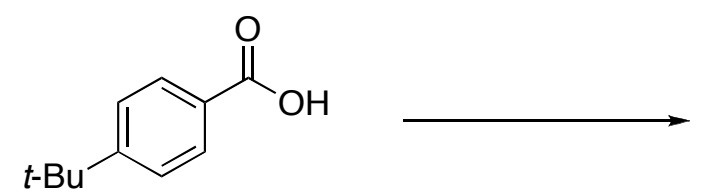

$\overbrace{t-\mathrm{Bu}}^{1}$

Benzyl 4-tert-butylphenylcarbamate (13). The title compound was prepared from 4-tert-butylbenzoic acid (178 $\mathrm{mg}, 1.00 \mathrm{mmol})$ according to the general procedure. The desired carbamate 13 (175 $\mathrm{mg}$, $62 \%$ ) was obtained after flash chromatography (10\% EtOAc/hexanes), as a white solid. $\mathrm{R}_{f} 0.40(20 \%$ EtOAc/hexanes); mp $64{ }^{\circ} \mathrm{C} ;{ }^{1} \mathrm{H}$ NMR $\left(400 \mathrm{MHz}, \mathrm{CDCl}_{3}\right.$ ) $\delta$ 7.43-7.33 (m, 9H), 6.71 (s (br), 1H), 5.21 (s, 2H), 1.31 (s (br), 9H); $\left.{ }^{13} \mathrm{C} \mathrm{NMR} \mathrm{(100} \mathrm{MHz,} \mathrm{CDCl}_{3}\right) \delta 153.3,146.3,136.1,135.0,128.6$ (2C), 128.3, 125.8, 118.4, 66.9, 34.2, 31.3; IR 3335, 3051, 2955, 2900, 2865, 1694, 1596, 1533, 1410, 1229, 1065, 831, 740, $661 \mathrm{~cm}^{-1}$; HMRS (ESI) calcd for $\mathrm{C}_{18} \mathrm{H}_{22} \mathrm{NO}_{2}[\mathrm{M}+\mathrm{H}]^{+}:$284.1651. Found: 284.1645.<smiles>O=C(O)c1ccc([N+](=O)[O-])cc1</smiles><smiles>C=CCOC(=O)Nc1ccc([N+](=O)[O-])cc1</smiles>

Allyl 4-nitrophenylcarbamate (14). The title compound was prepared from 4-nitrobenzoic acid (167 $\mathrm{mg}, 1.00 \mathrm{mmol})$ according to the general procedure. The desired carbamate $14(173 \mathrm{mg}, 78 \%)$ was obtained as a yellow solid after flash chromatography $\left(30 \%\right.$ EtOAc/hexanes). $\mathrm{R}_{f} \quad 0.16$ (20\% EtOAc/hexanes); mp $94{ }^{\circ} \mathrm{C} ;{ }^{1} \mathrm{H}$ NMR $\left(400 \mathrm{MHz} \mathrm{CDCl}_{3}\right) \delta 8.19(\mathrm{~d}, J=9 \mathrm{~Hz}, 2 \mathrm{H}), 7.58-7.55(\mathrm{~m}, 2 \mathrm{H})$, 7.20 (s (br), 1H), 6.00-5.90 (m, 1H), 5.37 (d, $J=17 \mathrm{~Hz}, 1 \mathrm{H}), 5.29$ (d, $J=10 \mathrm{~Hz}, 1 \mathrm{H}), 4.69$ (d, $J=6 \mathrm{~Hz}$, $2 \mathrm{H}) ;{ }^{13} \mathrm{C} \mathrm{NMR}\left(100 \mathrm{MHz}, \mathrm{CDCl}_{3}\right) \delta 152.6,143.9,142.9,131.7,125.2,118.9,117.7,66.4$; IR 3376, 3092, 2947, 2887, 1731, 1546, 1508, 1493, 1321, 1204, 1056, 853, $747 \mathrm{~cm}^{-1}$; HMRS (ESI) calcd for $\mathrm{C}_{10} \mathrm{H}_{11} \mathrm{~N}_{2} \mathrm{O}_{4}[\mathrm{M}+\mathrm{H}]^{+}:$223.0713. Found: 223.0709.<smiles>CC(=O)c1ccc(C(=O)O)cc1</smiles>

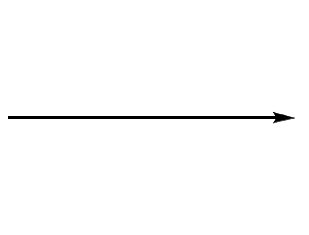<smiles>C=CCOC(=O)Nc1ccc(C(C)=O)cc1</smiles>

Allyl 4-acetylphenylcarbamate (15). The title compound was prepared from 4-acetylbenzoic acid (164 $\mathrm{mg}, 1.00 \mathrm{mmol})$ according to the general procedure. The desired carbamate $\mathbf{1 5}(144 \mathrm{mg}, 66 \%)$ was obtained as a white solid after flash chromatography (30\% EtOAc/hexanes). $\mathrm{R}_{f} \quad 0.11 \quad(20 \%$ EtOAc/hexanes); mp $116{ }^{\circ} \mathrm{C} ;{ }^{1} \mathrm{H}$ NMR $\left(400 \mathrm{MHz}, \mathrm{CDCl}_{3}\right) \delta 7.93(\mathrm{~d}, J=9 \mathrm{~Hz}, 2 \mathrm{H}), 7.50(\mathrm{~d}, J=9 \mathrm{~Hz}$, 2H), 7.09 (s (br), 1H), 5.94 (ddt, $J=17,10,6 \mathrm{~Hz}, 1 \mathrm{H}), 5.40-5.35$ (m, 1H), 5.29-5.26 (m, 1H), 4.69-4.67 $(\mathrm{m}, 2 \mathrm{H}), 2.57(\mathrm{~s}, 3 \mathrm{H}) ;{ }^{13} \mathrm{C}$ NMR $\left(100 \mathrm{MHz} \mathrm{CDCl}_{3}\right) \delta 197.1,152.8,142.5,131.98,131.95,129.8$, 118.5, 117.6, 66.0, 26.3 ; IR 3297, 3191, 3112, 3065, 2949, 1724, 1660, 1588, 1531, 1410, 1208, 1053, $845,697 \mathrm{~cm}^{-1}$; HMRS (ESI) calcd for $\mathrm{C}_{12} \mathrm{H}_{14} \mathrm{NO}_{3}[\mathrm{M}+\mathrm{H}]^{+}:$220.0968. Found: 220.0959 .

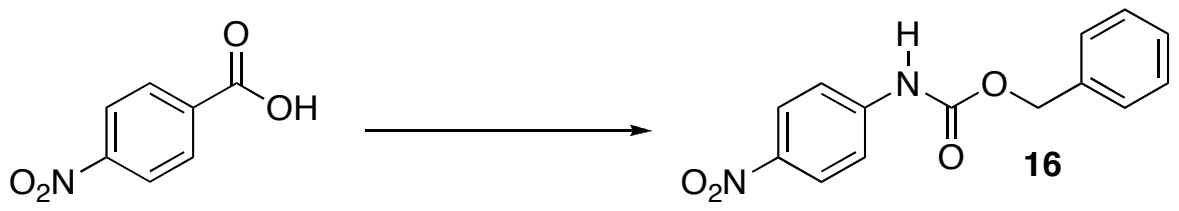

Benzyl 4-nitrophenylcarbamate (16). The title compound was prepared from 4-nitrobenzoic acid (167 $\mathrm{mg}, 1.00 \mathrm{mmol})$ according to the general procedure. The desired carbamate $16(228 \mathrm{mg}, 84 \%)$ was obtained after flash chromatography $\left(20 \%\right.$ EtOAc/hexanes), as a yellow solid. $\mathrm{R}_{f} 0.24(20 \%$ EtOAc/hexanes); mp $142{ }^{\circ} \mathrm{C} ;{ }^{1} \mathrm{H}$ NMR (400 MHz, DMSO) $\delta 10.52$ (s (br), 1H), 8.18 (d , J=9 Hz, 2H), $7.70(\mathrm{~d}, J=9 \mathrm{~Hz}, 2 \mathrm{H}), 7.46-7.33(\mathrm{~m}, 5 \mathrm{H}), 5.20(\mathrm{~s}, 2 \mathrm{H}) ;{ }^{13} \mathrm{C}$ NMR (100 MHz, DMSO) $\delta$ 153.0, 145.6, 
141.6, 136.0, 128.4, 128.3, 128.2, 125.0, 117.5, 66.4; IR 3330, 1738, 1599, 1544, 1492, 1328, 1213, 1061, 845, $727 \mathrm{~cm}^{-1}$; HMRS (ESI) calcd for $\mathrm{C}_{14} \mathrm{H}_{13} \mathrm{~N}_{2} \mathrm{O}_{4}[\mathrm{M}+\mathrm{H}]^{+}:$273.0870. Found: 273.0876.<smiles>CC(=O)c1ccc(C(=O)O)cc1</smiles><smiles>CC(=O)c1ccc(NC(=O)OCc2ccccc2)cc1</smiles>

Benzyl 4-acetylphenylcarbamate (17). The title compound was prepared from 4-acetylbenzoic acid (164 $\mathrm{mg}, 1.00 \mathrm{mmol})$ according to the general procedure. The desired carbamate $17(215 \mathrm{mg}, 80 \%)$ was obtained after flash chromatography $\left(30 \%\right.$ EtOAc/hexanes), as a white solid. $\mathrm{R}_{f} 0.12(20 \%$ EtOAc/hexanes); mp $114{ }^{\circ} \mathrm{C} ;{ }^{1} \mathrm{H}$ NMR $\left(400 \mathrm{MHz}, \mathrm{CDCl}_{3}\right) \delta 7.91(\mathrm{~d}, J=9 \mathrm{~Hz}, 2 \mathrm{H}), 7.51(\mathrm{~d}, J=9 \mathrm{~Hz}$,

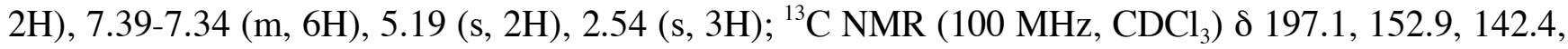
135.6, 132.0, 129.8, 128.6, 128.4, 128.2, 117.6, 67.2, 26.3; IR 3283, 3190, 3062, 2966, 1718, 1666, 1588, 1537, 1413, 1319, 1217, 1187, 1047, 959, 843, $695 \mathrm{~cm}^{-1}$; HMRS (ESI) calcd for $\mathrm{C}_{16} \mathrm{H}_{16} \mathrm{NO}_{3}$ $[\mathrm{M}+\mathrm{H}]^{+}:$270.1125. Found: 270.1124 .<smiles>O=C(O)c1cccc(Cl)c1</smiles><smiles>C=CCOC(=O)Nc1cccc(Cl)c1</smiles>

Allyl 3-chlorophenylcarbamate (18). The title compound was prepared from 3-chlorobenzoic acid (156 $\mathrm{mg}, 1.00 \mathrm{mmol})$ according to the general procedure. The desired carbamate $18(139 \mathrm{mg}, 66 \%)$ was obtained as a yellow oil after flash chromatography $\left(10 \%\right.$ EtOAc/hexanes). $\mathrm{R}_{f} \quad 0.38 \quad(20 \%$ EtOAc/hexanes); ${ }^{1} \mathrm{H}$ NMR (400 MHz, $\mathrm{CDCl}_{3}$ ) $\delta 7.54$ (s (br), 1H), 7.28-7.20 (m, 2H), 7.06-7.04 (m, $1 \mathrm{H}), 6.94$ (s (br), 1H), 6.02-5.92 (m, 1H), $5.38(\mathrm{~d}, J=17 \mathrm{~Hz}, 1 \mathrm{H}), 5.29(\mathrm{~d}, J=10 \mathrm{~Hz}, 1 \mathrm{H}), 4.69(\mathrm{~d}, J=$ $5 \mathrm{~Hz}, 2 \mathrm{H}) ;{ }^{13} \mathrm{C}$ NMR $\left(100 \mathrm{MHz}, \mathrm{CDCl}_{3}\right) \delta 153.0,138.9,134.7,132.1,129.9,123.5,118.7,118.4$, 116.6, 66.0; IR 3309, 3085, 1710, 1598, 1535, 1427, 1275, 1225, 1058, 879, $777 \mathrm{~cm}^{-1}$; HMRS (ESI) calcd for $\mathrm{C}_{10} \mathrm{H}_{11} \mathrm{ClNO}_{2}[\mathrm{M}+\mathrm{H}]^{+}: 212.0473$. Found: 212.0483 .<smiles>O=C(O)c1cccc(Br)c1</smiles><smiles>C=CCOC(=O)Nc1cccc(Br)c1</smiles>

Allyl 3-bromophenylcarbamate (19). The title compound was prepared from 3-bromobenzoic acid (201 $\mathrm{mg}, 1.00 \mathrm{mmol}$ ) according to the general procedure. The desired carbamate $19(133 \mathrm{mg}, 52 \%)$ was obtained as a colorless oil after flash chromatography (10\% EtOAc/hexanes). $\mathrm{R}_{f} 0.38 \quad(20 \%$ EtOAc/hexanes); ${ }^{1} \mathrm{H}$ NMR (400 MHz, $\mathrm{CDCl}_{3}$ ) $\delta 7.68$ (s (br), 1H), 7.31-7.28 (m, 1H), 7.23-7.16 (m, $2 \mathrm{H}), 6.72(\mathrm{~s}(\mathrm{br}), 1 \mathrm{H}), 5.98(\mathrm{ddt}, J=17,10,6 \mathrm{~Hz}, 1 \mathrm{H}), 5.40-5.36(\mathrm{~m}, 1 \mathrm{H}), 5.31-5.28(\mathrm{~m}, 1 \mathrm{H}), 4.70-4.68$ $(\mathrm{m}, 2 \mathrm{H}) ;{ }^{13} \mathrm{C}$ NMR $\left(100 \mathrm{MHz}, \mathrm{CDCl}_{3}\right) \delta 153.0,139.0,132.1,130.2,126.3,122.6,121.5,118.4,117.0$, 66.0 ; IR 3311, 3082, 2948, 1704, 1589, 1525, 1480, 1274, 1215, 1055, 994, $771 \mathrm{~cm}^{-1}$; HMRS (ESI) calcd for $\mathrm{C}_{10} \mathrm{H}_{11} \mathrm{NO}_{2} \mathrm{Br}[\mathrm{M}+\mathrm{H}]^{+}:$255.9969. Found: 255.9967.<smiles>O=C(O)c1cccc(Cl)c1</smiles><smiles>O=C(Nc1cccc(Cl)c1)OCc1ccccc1</smiles>

Benzyl 3-chlorophenylcarbamate (20). The title compound was prepared from 3-chlorobenzoic acid (156 $\mathrm{mg}, 1.00 \mathrm{mmol})$ according to the general procedure. The desired carbamate $20(175 \mathrm{mg}, 67 \%)$ 
was obtained as a colorless oil after flash chromatography (15\% EtOAc/hexanes). $\mathrm{R}_{f} 0.44$ (20\% EtOAc/hexanes); ${ }^{1} \mathrm{H}$ NMR (400 MHz, $\mathrm{CDCl}_{3}$ ) $\delta 7.53$ (s (br), 1H), 7.39-7.36 (m, 5H), 7.26-7.18 (m, $2 \mathrm{H}), 7.06-7.04(\mathrm{~m}, 2 \mathrm{H}), 5.20(\mathrm{~s}, 2 \mathrm{H}) ;{ }^{13} \mathrm{C} \mathrm{NMR}\left(100 \mathrm{MHz}, \mathrm{CDCl}_{3}\right) \delta$ 153.2, 138.9, 135.6, 134.5, 129.9, 128.5, 128.3, 128.2, 123.4, 118.6, 116.6, 67.1; IR 3310, 3033, 2955, 1704, 1594, 1530, 1426, 1213, $1054,883,697 \mathrm{~cm}^{-1}$; HMRS (ESI) calcd for $\mathrm{C}_{14} \mathrm{H}_{13} \mathrm{NO}_{2} \mathrm{Cl}[\mathrm{M}+\mathrm{H}]^{+}:$262.0632. Found: 262.0629.<smiles>O=C(O)c1cccc(Br)c1</smiles><smiles>O=C(Nc1cccc(Br)c1)OCc1ccccc1</smiles>

Benzyl 3-bromophenylcarbamate (21). The title compound was prepared from 3-bromobenzoic acid (201 $\mathrm{mg}, 1.00 \mathrm{mmol})$ according to the general procedure. The desired carbamate $21(220 \mathrm{mg}, 72 \%)$ was obtained as a colorless oil after flash chromatography (15\% EtOAc/hexanes). $\mathrm{R}_{f} 0.28$ (20\% EtOAc/hexanes); ${ }^{1} \mathrm{H}$ NMR (400 MHz, $\mathrm{CDCl}_{3}$ ) $\delta 7.69$ (s (br), 1H), 7.40-7.39 (m, 5H), 7.32-7.14 (m, $3 \mathrm{H}), 7.07(\mathrm{~s}(\mathrm{br}), 1 \mathrm{H}), 5.22(\mathrm{~s}, 2 \mathrm{H}) ;{ }^{13} \mathrm{C} \mathrm{NMR}\left(100 \mathrm{MHz}, \mathrm{CDCl}_{3}\right) \delta 153.1,139.0,135.6,130.2,128.5$, 128.3, 128.2, 126.3, 122.6, 121.5, 117.1, 67.1; IR 3310, 3032, 2955, 1700, 1588, 1522, 1421, 1274, 1211, 1051, 869, $769 \mathrm{~cm}^{-1}$; HMRS (ESI) calcd for $\mathrm{C}_{14} \mathrm{H}_{13} \mathrm{NO}_{2} \mathrm{Br}[\mathrm{M}+\mathrm{H}]^{+}:$306.0120. Found: 306.0124.<smiles>O=C(O)c1ccccc1Br</smiles><smiles>C=CCOC(=O)Nc1ccccc1Br</smiles>

Allyl 2-bromophenylcarbamate (22). The title compound was prepared from 2-bromobenzoic acid (201 $\mathrm{mg}, 1.00 \mathrm{mmol}$ ) according to the general procedure. The desired carbamate $\mathbf{2 2}(212 \mathrm{mg}, 83 \%)$ was obtained as a colorless oil after flash chromatography (10\% EtOAc/hexanes). $\mathrm{R}_{f} 0.55$ (20\% EtOAc/hexanes); ${ }^{1} \mathrm{H}$ NMR $\left(400 \mathrm{MHz}, \mathrm{CDCl}_{3}\right) \delta 8.15(\mathrm{~d}, J=8 \mathrm{~Hz}, 1 \mathrm{H}), 7.51(\mathrm{~d}, J=8 \mathrm{~Hz}, 1 \mathrm{H}), 7.30$ (t, $J=8 \mathrm{~Hz}, 1 \mathrm{H}), 7.19(\mathrm{~s}(\mathrm{br}), 1 \mathrm{H}), 6.92(\mathrm{t}, J=8 \mathrm{~Hz}, 1 \mathrm{H}), 5.99$ (ddt, $J=17,10,6 \mathrm{~Hz}, 1 \mathrm{H}), 5.40-5.36$ (m, $1 \mathrm{H})$, 5.30-5.27 (m, 1H), 4.70-4.68 (m, 2H); ${ }^{13} \mathrm{C}$ NMR (100 MHz, $\left.\mathrm{CDCl}_{3}\right) \delta 152.7,135.6,132.2,132,1$, 128.3, 124.2, 120.1, 118.4, 112.5, 66.0; IR 3404, 2947, 1739, 1593, 1578, 1521, 1437, 1207, 1065, 750 $\mathrm{cm}^{-1}$; HMRS (ESI) calcd for $\mathrm{C}_{10} \mathrm{H}_{11} \mathrm{NO}_{2}[\mathrm{M}+\mathrm{H}]^{+}: 255.9966$. Found: 255.9967.<smiles>O=C(O)c1ccccc1I</smiles><smiles>C=CCOC(=O)Nc1ccccc1I</smiles>

Allyl 2-iodophenylcarbamate (23). The title compound was prepared from 2-iodobenzoic acid (248 $\mathrm{mg}, 1.00 \mathrm{mmol})$ according to the general procedure. The desired carbamate $\mathbf{2 3}(218 \mathrm{mg}, 72 \%)$ was obtained as a colorless oil after flash chromatography (5\% EtOAc/hexanes). $\mathrm{R}_{f} 0.61$ (20\% EtOAc/hexanes); ${ }^{1} \mathrm{H}$ NMR (400 MHz, $\left.\mathrm{CDCl}_{3}\right) \delta 8.07$ (d , $\left.J=8 \mathrm{~Hz}, 1 \mathrm{H}\right), 7.77$ (d, $\left.J=8 \mathrm{~Hz}, 1 \mathrm{H}\right), 7.35$ (t, $J=8 \mathrm{~Hz}, 1 \mathrm{H}), 7.03(\mathrm{~s}(\mathrm{br}), 1 \mathrm{H}), 6.82(\mathrm{t}, J=8 \mathrm{~Hz}, 1 \mathrm{H}), 6.02$ (ddt, $J=17,10,6 \mathrm{~Hz}, 1 \mathrm{H}), 5.43-5.38(\mathrm{~m}$, $1 \mathrm{H}), 5.33-5.29(\mathrm{~m}, 1 \mathrm{H}), 4.72-4.70(\mathrm{~m}, 2 \mathrm{H}) ;{ }^{13} \mathrm{C}$ NMR $\left(100 \mathrm{MHz}, \mathrm{CDCl}_{3}\right) \delta 153.0,138.8,138.2,132.2$, 129.2, 125.1, 120.3, 118.5, 88.8, 66.1; IR 3385, 2959, 1733, 1585, 1516, 1434, 1204, 1038, $750 \mathrm{~cm}^{-1}$; HMRS (ESI) calcd for $\mathrm{C}_{10} \mathrm{H}_{11} \mathrm{I} \mathrm{NO} \mathrm{N}_{2}[\mathrm{M}+\mathrm{H}]^{+}$: 303.9829. Found: 303.9826 . 
<smiles>O=C(O)c1ccccc1Br</smiles><smiles>O=C(Nc1ccccc1Br)OCc1ccccc1</smiles>

Benzyl 2-bromophenylcarbamate (24). The title compound was prepared from 2-bromobenzoic acid (201 $\mathrm{mg}, 1.00 \mathrm{mmol})$ according to the general procedure. The desired carbamate $24(278 \mathrm{mg}, 91 \%)$ was obtained as a white solid, after flash chromatography (10\% EtOAc/hexanes). $\mathbf{R}_{f} 0.45 \quad(20 \%$ EtOAc/hexanes); mp $41{ }^{\circ} \mathrm{C} ;{ }^{1} \mathrm{H}$ NMR $\left(400 \mathrm{MHz} \mathrm{CDCl}_{3}\right) \delta 8.18(\mathrm{~d}, J=8 \mathrm{~Hz}, 1 \mathrm{H}), 7.51(\mathrm{~d}, J=8 \mathrm{~Hz}$, $1 \mathrm{H}), 7.45-7.29(\mathrm{~m}, 6 \mathrm{H}), 7.22(\mathrm{~s}(\mathrm{br}), 1 \mathrm{H}), 6.94(\mathrm{t}, J=8 \mathrm{~Hz}, 1 \mathrm{H}), 5.23(\mathrm{~s}, 2 \mathrm{H}) ;{ }^{13} \mathrm{C} \mathrm{NMR}(100 \mathrm{MHz}$, $\left.\mathrm{CDCl}_{3}\right) \delta$ 153.0, 135.7, 132.3, 128.7, 128.5, 128.42, 128.38, 124.3, 120.1, 112.5, 67.3; IR 3288, 3056, 3031, 2954, 1698, 1579, 1522, 1437, 1233, 1068, 1025, 843, $738 \mathrm{~cm}^{-1}$; HMRS (ESI) calcd for $\mathrm{C}_{14} \mathrm{H}_{13} \mathrm{NO}_{2} \mathrm{Br}[\mathrm{M}+\mathrm{H}]^{+}:$306.0124. Found: 306.0124.<smiles>O=C(O)c1ccccc1I</smiles><smiles>O=C(Nc1ccccc1I)OCc1ccccc1</smiles>

Benzyl 2-iodophenylcarbamate (25). The title compound was prepared from 2-iodobenzoic acid (248 $\mathrm{mg}, 1.00 \mathrm{mmol})$ according to the general procedure. The desired carbamate $25(328 \mathrm{mg}$, 93\%) was obtained as a white solid, after flash chromatography (20\% EtOAc/hexanes). $\mathrm{R}_{f} \quad 0.57 \quad(20 \%$ EtOAc/hexanes); mp $49{ }^{\circ} \mathrm{C} ;{ }^{1} \mathrm{H}$ NMR $\left(400 \mathrm{MHz}, \mathrm{CDCl}_{3}\right) \delta 8.10(\mathrm{~d}, J=8 \mathrm{~Hz}, 1 \mathrm{H}), 7.77(\mathrm{~d}, J=8 \mathrm{~Hz}$, $1 \mathrm{H}), 7.46-7.33(\mathrm{~m}, 6 \mathrm{H}), 7.05(\mathrm{~s}(\mathrm{br}), 1 \mathrm{H}), 6.81(\mathrm{t}, J=8 \mathrm{~Hz}, 1 \mathrm{H}), 5.24(\mathrm{~s}, 2 \mathrm{H}) ;{ }^{13} \mathrm{C}$ NMR $(100 \mathrm{MHz}$, $\left.\mathrm{CDCl}_{3}\right) \delta 153.2,151.4,138.8,138.2,135.7,129.2,128.6,128.3,125.1,120.3,88.8,67.2$; IR 3383, 3031, 2953, 1732, 1586, 1514, 1432, 1201, 1036, $741 \mathrm{~cm}^{-1}$; HMRS (ESI) calcd for $\mathrm{C}_{14} \mathrm{H}_{12} \mathrm{INO}_{2}$ $[\mathrm{M}+\mathrm{H}]^{+}:$353.9985. Found: 353.9982 .<smiles>O=C(O)c1ccccc1I</smiles><smiles>O=C(Nc1ccccc1I)OCC(Cl)(Cl)Cl</smiles>

2,2,2-Trichloroethyl 2-iodophenylcarbamate (26). The title compound was prepared from 2-iodobenzoic acid (248 $\mathrm{mg}, 1.00 \mathrm{mmol}$ ) according to the general procedure. The desired carbamate $\mathbf{2 6}$ (280 mg, 71\%) was obtained as a white solid, after flash chromatography (5\% EtOAc/hexanes). $\mathrm{R}_{f} 0.82$ (20\% EtOAc/hexanes); mp $61{ }^{\circ} \mathrm{C} ;{ }^{1} \mathrm{H}$ NMR $\left(400 \mathrm{MHz}, \mathrm{CDCl}_{3}\right) \delta 7.99$ (d (br), $\left.J=7 \mathrm{~Hz}, 1 \mathrm{H}\right), 7.78$ (d, $J$ $=8 \mathrm{~Hz}, 1 \mathrm{H}), 7.35(\mathrm{t}, J=8 \mathrm{~Hz}, 1 \mathrm{H}), 7.13(\mathrm{~s}(\mathrm{br}), 1 \mathrm{H}), 6.84(\mathrm{t}, J=7 \mathrm{~Hz}, 1 \mathrm{H}), 4.83(\mathrm{~s}, 2 \mathrm{H}) ;{ }^{13} \mathrm{C} \mathrm{NMR}(100$ $\left.\mathrm{MHz}, \mathrm{CDCl}_{3}\right) \delta$ 151.9, 139.0, 137.5, 129.4, 125.9, 120.8, 95.1, 74.6; IR 3295, 3057, 2958, 1762, 1691, 1522, 1435, 1279, 1226, 1102, 990, 808, $712 \mathrm{~cm}^{-1}$; HMRS (ESI) calcd for $\mathrm{C}_{9} \mathrm{H}_{7} \mathrm{Cl}_{3} \mathrm{INO}_{2}[\mathrm{M}+\mathrm{H}]^{+}$: 393.8659. Found: 393.8653.<smiles>C=CCOC(=O)Nc1cc(S(C)(=O)=O)ccc1Cl</smiles>

Allyl 2-chloro-5-(methylthio)phenylcarbamate (27). The title compound was prepared from 2-chloro-5-(methylthio)benzoic acid $(203 \mathrm{mg}, 1.00 \mathrm{mmol}$ ) according to the general procedure. The desired carbamate $27(211 \mathrm{mg}, 82 \%)$ was obtained as a colorless oil after flash chromatography (10\% EtOAc/hexanes). $\mathrm{R}_{f} 0.50$ (20\% EtOAc/hexanes); ${ }^{1} \mathrm{H}$ NMR (400 MHz, $\mathrm{CDCl}_{3}$ ) $\delta 8.14$ (s (br), 1H), 7.24$7.21(\mathrm{~m}, 2 \mathrm{H}), 6.87(\mathrm{~d}, J=8 \mathrm{~Hz}, 1 \mathrm{H}), 6.04-5.95(\mathrm{~m}, 1 \mathrm{H}), 5.40(\mathrm{~d}, J=17 \mathrm{~Hz}, 1 \mathrm{H}), 5.30(\mathrm{~d}, J=10 \mathrm{~Hz}$, 
$1 \mathrm{H}), 4.70(\mathrm{~d}, J=5 \mathrm{~Hz}, 2 \mathrm{H}), 2.49(\mathrm{~s}, 3 \mathrm{H}) ;{ }^{13} \mathrm{C} \mathrm{NMR}\left(100 \mathrm{MHz}, \mathrm{CDCl}_{3}\right) \delta 152.6,138.8,134.7,131.9$, 128.8, 121.3, 118.5, 118.1, 116.6, 66.1, 15.6; IR 3413, 2922, 1739, 1577, 1516, 1407, 1272, 1209, 1067, 928, $765 \mathrm{~cm}^{-1}$; HMRS (ESI) calcd for $\mathrm{C}_{11} \mathrm{H}_{13} \mathrm{ClNO}_{2} \mathrm{~S}[\mathrm{M}+\mathrm{H}]^{+}:$258.0350. Found: 258.0351.<smiles>CSc1ccc(Cl)c(C(=O)O)c1</smiles><smiles>CSc1ccc(Cl)c(NC(=O)OCc2ccccc2)c1</smiles>

Benzyl 2-chloro-5-(methylthio)phenylcarbamate (28). The title compound was prepared from 2-chloro-5-(methylthio)benzoic acid $(203 \mathrm{mg}, 1.00 \mathrm{mmol}$ ) according to the general procedure. The desired carbamate 28 (249 mg, 81\%) was obtained as a white solid, after flash chromatography (10\% EtOAc/hexanes). $\mathrm{R}_{f} 0.49$ (20\% EtOAc/hexanes); mp $42{ }^{\circ} \mathrm{C} ;{ }^{1} \mathrm{H}$ NMR (400 MHz, $\mathrm{CDCl}_{3}$ ) $\delta 8.17$ (s (br), $1 \mathrm{H}), 7.45-7.35(\mathrm{~m}, 5 \mathrm{H}), 7.23(\mathrm{~d}, J=8 \mathrm{~Hz}, 2 \mathrm{H}), 6.88(\mathrm{~d}, J=8 \mathrm{~Hz}, 1 \mathrm{H}), 5.24(\mathrm{~s}, 2 \mathrm{H}), 2.49(\mathrm{~s}, 3 \mathrm{H}) ;{ }^{13} \mathrm{C}$ NMR $\left(100 \mathrm{MHz}, \mathrm{CDCl}_{3}\right) \delta 152.7,138.7,135.5,134.7,128.8,128.5,128.4,128.3,121.3,118.1,116.6$, 67.3, 15.5; IR 3408, 2921, 1737, 1577, 1515, 1407, 1206, 1063, 905, $697 \mathrm{~cm}^{-1}$; HMRS (ESI) calcd for $\mathrm{C}_{15} \mathrm{H}_{15} \mathrm{NO}_{2} \mathrm{SCl}[\mathrm{M}+\mathrm{H}]^{+}: 308.0518$. Found: 308.0506.<smiles>O=C(O)c1cc2ccccc2o1</smiles><smiles>C=CCOC(=O)Nc1cc2ccccc2o1</smiles>

Allyl benzofuran-2-ylcarbamate (29). The title compound was prepared from benzofuranbenzoic acid (162 $\mathrm{mg}, 1.00 \mathrm{mmol})$ according to the general procedure. The desired carbamate $29(150 \mathrm{mg}, 69 \%)$ was obtained as a yellow solid after flash chromatography $\left(20 \%\right.$ EtOAc/hexanes). $\mathrm{R}_{f} 0.30 \quad(20 \%$ EtOAc/hexanes); mp $94{ }^{\circ} \mathrm{C} ;{ }^{1} \mathrm{H}$ NMR $\left(400 \mathrm{MHz}, \mathrm{CDCl}_{3}\right) \delta$ 7.45-7.47 (m, 1H), 7.34-7.33 (m, 1H), 7.22-7.15 (m, 3H), 6.52 (s (br), $1 \mathrm{H}), 5.98$ (ddt, $J=17,10,6 \mathrm{~Hz}, 1 \mathrm{H}), 5.42-5.37(\mathrm{~m}, 1 \mathrm{H}), 5.32-5.29(\mathrm{~m}$, $1 \mathrm{H}), 4.75-4.73(\mathrm{~m}, 2 \mathrm{H}) ;{ }^{13} \mathrm{C} \mathrm{NMR}\left(100 \mathrm{MHz}, \mathrm{CDCl}_{3}\right) \delta 151.7,150.0,147.7,131.7,129.4,123.1,122.5$, 120.0, 118.8, 110.2, 88.9, 66.7; IR 3237, 3073, 2962, 1703, 1607, 1551, 1454, 1226, 1061, 938, 783 $\mathrm{cm}^{-1}$; HMRS (ESI) calcd for $\mathrm{C}_{12} \mathrm{H}_{12} \mathrm{NO}_{3}[\mathrm{M}+\mathrm{H}]^{+}: 218.0812$. Found: 218.0817 .<smiles>O=C(O)c1cc2ccccc2o1</smiles><smiles>O=C(Nc1cc2ccccc2o1)OCc1ccccc1</smiles>

Benzyl benzofuran-2-ylcarbamate (30). The title compound was prepared from 2-benzofuranbenzoic acid $(162 \mathrm{mg}, 1.00 \mathrm{mmol})$ according to the general procedure. The desired carbamate $30(214 \mathrm{mg}$, $80 \%$ ) was obtained as a yellow solid, after flash chromatography $\left(20 \%\right.$ EtOAc/hexanes). $\mathrm{R}_{f} 0.41(20 \%$ EtOAc/hexanes); mp $81{ }^{\circ} \mathrm{C} ;{ }^{1} \mathrm{H}$ NMR $\left(400 \mathrm{MHz}, \mathrm{CDCl}_{3}\right.$ ) $\delta$ 7.47-7.32 (m, 7H), 7.28 (s (br), 1H), 7.237.15 (m, 2H), 6.55 (s (br), 1H), 5.27 (s, 2H); ${ }^{13} \mathrm{C} \mathrm{NMR} \mathrm{(100} \mathrm{MHz,} \mathrm{CDCl}_{3}$ ) $\delta 151.8,150.1,147.6,135.3$, 129.4, 128.65, 128.57, 128.4, 123.2, 122.5, 120.1, 110.2, 88.9, 67.9; IR 3287, 3149, 3063, 2962, 1703, $1608,1542,1454,1215,1058,733 \mathrm{~cm}^{-1}$; HMRS (ESI) calcd for $\mathrm{C}_{16} \mathrm{H}_{14} \mathrm{NO}_{3}[\mathrm{M}+\mathrm{H}]^{+}:$268.0979. Found: 268.0968 .<smiles>C=CCOC(=O)N/C=C/c1ccccc1</smiles>

Allyl styrylcarbamate (31). The title compound was prepared from trans-cinamic acid (148 mg, 1.00 $\mathrm{mmol}$ ) according to the general procedure. The desired carbamate 31 (106 $\mathrm{mg}, 52 \%$ ) was obtained as a 
white solid after flash chromatography (20\% EtOAc/hexanes). $\mathrm{R}_{f} 0.40$ (20\% EtOAc/hexanes); mp $68{ }^{\circ} \mathrm{C}$; ${ }^{1} \mathrm{H}$ NMR $\left(400 \mathrm{MHz}, \mathrm{DMSO}, 85^{\circ} \mathrm{C}\right) \delta 9.45$ (s (br), 1H), 7.29-7.24 (m, 4H), 7.14-7.08 (m, 2H), $6.11(\mathrm{~d}, J=14 \mathrm{~Hz}, 1 \mathrm{H}), 5.97$ (ddt, $J=17,10,6 \mathrm{~Hz}, 1 \mathrm{H}), 5.37-5.32(\mathrm{~m}, 1 \mathrm{H}), 5.25-5.22(\mathrm{~m}, 1 \mathrm{H}) 4.62-$ $4.60(\mathrm{~m}, 2 \mathrm{H}) ;{ }^{13} \mathrm{C} \mathrm{NMR}\left(100 \mathrm{MHz}, \mathrm{CDCl}_{3}\right) \delta 153.3,136.1,132.1,128.6,126.3,125.3,123.9,118.4$, 110.8, 66.2 ; IR 3291, 3060, 3027, 1698, 1656, 1530, 1252, 1055, 937, 748, $690 \mathrm{~cm}^{-1}$; HMRS (ESI) calcd for $\mathrm{C}_{12} \mathrm{H}_{13} \mathrm{NO}_{2}[\mathrm{M}+\mathrm{H}]^{+}:$204.1019. Found: 204.1017.<smiles>O=C(O)/C=C/c1ccccc1</smiles><smiles>O=C(N/C=C/c1ccccc1)OCc1ccccc1</smiles>

Benzyl styrylcarbamate (32). The title compound was prepared from trans-cinnamic acid (148 $\mathrm{mg}$, $1.00 \mathrm{mmol})$ according to the general procedure. The desired carbamate $32(129 \mathrm{mg}, 51 \%)$ was obtained as a white solid after flash chromatography (20\% EtOAc/hexanes). $\mathrm{R}_{f} 0.42$ (20\% EtOAc/hexanes); $\mathrm{mp}$ $94{ }^{\circ} \mathrm{C} ;{ }^{1} \mathrm{H}$ NMR $\left(400 \mathrm{MHz}, \mathrm{DMSO}, 85^{\circ} \mathrm{C}\right) \delta 9.51$ (s (br), 1H), 7.41-7.32 (m, 4H), 7.29-7.24 (m, 5H), 7.15-7.09 (m, 2H), $6.11(\mathrm{~d}, J=14 \mathrm{~Hz}, 1 \mathrm{H}), 5.16(\mathrm{~s}, 2 \mathrm{H}) ;{ }^{13} \mathrm{C} \mathrm{NMR}\left(100 \mathrm{MHz}, \mathrm{CDCl}_{3}\right) \delta 153.4,136.1$, 135.7, 128.6 (2C), 128.4, 128.3, 126.3, 125.2, 123.8, 110.9, 67.4; IR 3276, 3032, 1710, 1659, 1517, 1496, 1221, 1051, 946, 746, $695 \mathrm{~cm}^{-1}$; HMRS (ESI) calcd for $\mathrm{C}_{16} \mathrm{H}_{16} \mathrm{NO}_{2}[\mathrm{M}+\mathrm{H}]^{+}:$254.1185. Found: 254.1175 .

\section{General procedure for the synthesis of aromatic ureas.}

To a solution of sodium azide $(0.110 \mathrm{~g}, 1.70 \mathrm{mmol})$, sodium tert-butoxide $(14.4 \mathrm{mg}, 0.150 \mathrm{mmol})$, and carboxylic acid $(1.00 \mathrm{mmol})$ in DME $(10.0 \mathrm{~mL})$ at $25^{\circ} \mathrm{C}$, was added the phenylchloroformate $(140 \mu \mathrm{L}$, $1.10 \mathrm{mmol})$. The resulting mixture was stirred at room temperature for 8 hours. The amine derivative $(1.50 \mathrm{mmol})$ was then added and the reaction mixture was stirred at for 16 hours. The reaction mixture was cooled to room temperature and diluted with hexane $(40 \mathrm{~mL})$. The resulting solution is poured into ice-cold water with continuous stirring, $10 \mathrm{~mL}$ water was added and the stirring maintains during 20 minutes. The solid was filtered and recrystallized when needed.

\section{Characterization of aromatic ureas.}

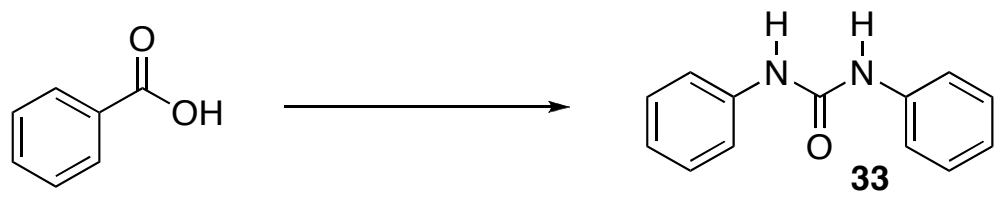

1,3-Diphenylurea (33). The title compound was prepared from benzoic acid (122 mg, $1.00 \mathrm{mmol})$ and aniline $(137 \mu \mathrm{L}, 1.50 \mathrm{mmol})$ according to the general procedure. The desired urea $33(189 \mathrm{mg}, 89 \%)$ was obtained as a white solid after filtration. $\mathrm{R}_{f} 0.51$ (40\% EtOAc/hexanes); mp $219{ }^{\circ} \mathrm{C} ;{ }^{1} \mathrm{H}$ NMR (400 MHz, DMSO) $\delta 8.24$ (s (br), 2H), 7.45 (d, $J=8 \mathrm{~Hz}, 4 \mathrm{H}), 7.27$ (t, $J=8 \mathrm{~Hz}, 4 \mathrm{H}), 6.96$ (t, $J=7 \mathrm{~Hz}, 2 \mathrm{H})$; ${ }^{13} \mathrm{C}$ NMR (100 MHz, DMSO) $\delta$ 152.4, 139.6, 128.7, 121.7, 118.1; IR 3281, 3036, 1646, 1592, 1538, 1496, 1439, 1230, 893, $751 \mathrm{~cm}^{-1}$; HMRS (ESI) calcd for $\mathrm{C}_{13} \mathrm{H}_{12} \mathrm{~N}_{2} \mathrm{ONa}[\mathrm{M}+\mathrm{Na}]^{+}:$235.0842. Found: 235.0843 . 
<smiles>O=C(O)c1ccc([N+](=O)[O-])cc1</smiles><smiles>O=C(Nc1ccccc1)Nc1ccc([N+](=O)[O-])cc1</smiles>

1-(4-Nitrophenyl)-3-phenylurea (34). The title compound was prepared from 4-nitrobenzoic acid (167 $\mathrm{mg}, 1.00 \mathrm{mmol})$ and aniline $(137 \mu \mathrm{L}, 1.50 \mathrm{mmol})$ according to the general procedure. The desired urea $34(182 \mathrm{mg}, 71 \%)$ was obtained as a pale yellow solid after filtration. $\mathrm{R}_{f} 0.34$ (40\% EtOAc/hexanes); mp $217{ }^{\circ} \mathrm{C}$; ${ }^{1} \mathrm{H}$ NMR (400 MHz, DMSO) $\delta 9.43$ (s (br), 1H), 8.91 (s (br), 1H), 8.19 (d, J=9 Hz, 2H), $7.69(\mathrm{~d}, J=9 \mathrm{~Hz}, 2 \mathrm{H}), 7.47(\mathrm{~d}, J=8 \mathrm{~Hz}, 2 \mathrm{H}), 7.31(\mathrm{t}, J=8 \mathrm{~Hz}, 2 \mathrm{H}), 7.02(\mathrm{t}, J=7 \mathrm{~Hz}, 1 \mathrm{H}) ;{ }^{13} \mathrm{C}$ NMR (100 MHz, DMSO) $\delta 151.9,146.3,140.9,138.9,128.8,125.1,122.5,118.6,117.4$; IR 3288, 164, $1606,1593,1556,1488,1343,1233,1110,856,750 \mathrm{~cm}^{-1}$; HMRS (ESI) calcd for $\mathrm{C}_{13} \mathrm{H}_{12} \mathrm{~N}_{3} \mathrm{O}_{3}[\mathrm{M}+\mathrm{H}]^{+}$: 258.0872. Found: 258.0872.<smiles>O=C(Nc1ccccc1I)Nc1ccccc1I</smiles>

1-(2-Iodophenyl)-3-phenylurea (35). The title compound was prepared from 2-iodobenzoic acid (248 $\mathrm{mg}, 1.00 \mathrm{mmol})$ and aniline $(137 \mu \mathrm{L}, 1.50 \mathrm{mmol})$ according to the general procedure. The desired urea 35 (200 mg, 59\%) was obtained as a white solid after filtration. $\mathrm{R}_{f} 0.64$ (40\% EtOAc/hexanes); mp 217 ${ }^{\circ} \mathrm{C}$; ${ }^{1} \mathrm{H}$ NMR (400 MHz, DMSO) $\delta 9.42$ (s (br), 1H), 7.88 (s (br), 1H), 7.85-7.82 (m, 2H), 7.47-7.45 $(\mathrm{m}, 2 \mathrm{H}), 7.36-7.27(\mathrm{~m}, 3 \mathrm{H}), 6.98(\mathrm{t}, J=7 \mathrm{~Hz}, 1 \mathrm{H}), 6.84(\mathrm{t}, J=7 \mathrm{~Hz}, 1 \mathrm{H}) ;{ }^{13} \mathrm{C}$ NMR $(100 \mathrm{MHz}, \mathrm{DMSO})$ $\delta 152.3,139.8,139.5,138.9,128.8,128.5,125.0,123.0,122.0,118.1,91.3$; IR 3280, 1645, 1573, 1543, $1444,1289,1229,1014,897,749 \mathrm{~cm}^{-1}$; HMRS (ESI) calcd for $\mathrm{C}_{13} \mathrm{H}_{12} \mathrm{~N}_{2} \mathrm{O} \mathrm{I}[\mathrm{M}+\mathrm{H}]^{+}: 338.9994$. Found: 338.9988<smiles>Cc1cc(C)c(NC(=O)Nc2ccccc2)c(C)c1</smiles>

1-Mesityl-3-phenylurea (36). The title compound was prepared from benzoic acid (122 mg, 1.00 $\mathrm{mmol})$ and mesidine $(211 \mu \mathrm{L}, 1.50 \mathrm{mmol})$ according to the general procedure. The desired urea 36 (203 $\mathrm{mg}, 80 \%$ ) was obtained as a white solid after trituration in $\mathrm{Et}_{2} \mathrm{O} . \mathrm{R}_{f} 0.52$ (40\% EtOAc/hexanes); mp 226 ${ }^{\circ} \mathrm{C}$; ${ }^{1} \mathrm{H}$ NMR (400 MHz, DMSO) $\delta 8.66$ (s (br), 1H), 7.59 (s, 1H), 7.44-7.42 (m, 2H), 7.25-7.22 (m,

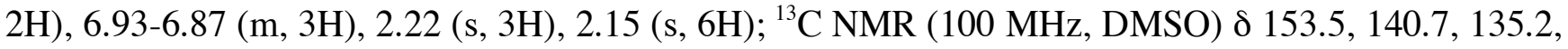
134.6, 132.9, 128.6, 128.2, 120.9, 117.5, 20.5, 18.3; IR 3279, 2917, 1641, 1595, 1550, 1495, 1228, 858, $694 \mathrm{~cm}^{-1}$; HMRS (ESI) calcd for $\mathrm{C}_{16} \mathrm{H}_{19} \mathrm{~N}_{2} \mathrm{O}[\mathrm{M}+\mathrm{H}]^{+}:$255.1492. Found: 255.1484.
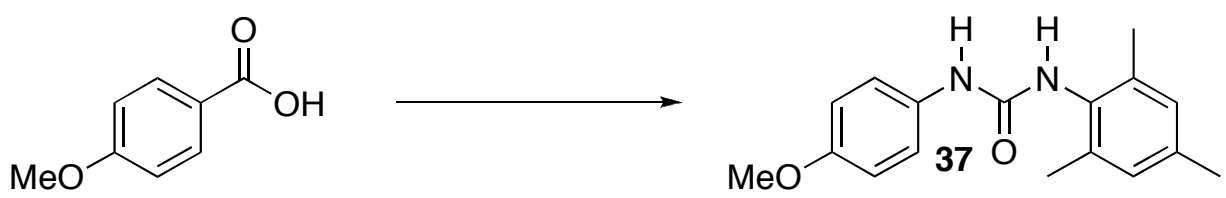

1-Mesityl-3-(4-methoxyphenyl)urea (37). The title compound was prepared from 4-methoxybenzoic acid $(152 \mathrm{mg}, 1.00 \mathrm{mmol})$ and mesidine $(211 \mu \mathrm{L}, 1.50 \mathrm{mmol})$ according to the general procedure. The desired urea $37(173 \mathrm{mg}, 61 \%)$ was obtained as a white solid after trituration in $\mathrm{Et}_{2} \mathrm{O} . \mathrm{R}_{f} 0.47(40 \%$ EtOAc/hexanes); mp $244{ }^{\circ} \mathrm{C}$; ${ }^{1} \mathrm{H}$ NMR (400 MHz, DMSO) $\delta 8.43$ (s (br), 1H), 7.50 (s, 1H), 7.33 (d, $J$ $=9 \mathrm{~Hz}, 2 \mathrm{H}), 6.87-6.82(\mathrm{~m}, 4 \mathrm{H}), 3.69(\mathrm{~s}, 3 \mathrm{H}), 2.22(\mathrm{~s}, 3 \mathrm{H}), 2.15(\mathrm{~s}, 6 \mathrm{H}) ;{ }^{13} \mathrm{C}$ NMR $(100 \mathrm{MHz}, \mathrm{DMSO})$ $\delta 154.0,153.4,135.2,134.7,133.4,132.7,128.2,119.5,113.8,55.1,20.4,18.1$; IR 3288, 2916, 2835, 
1637, 1599, 15554, 1508, 1247, 1222, 837, $683 \mathrm{~cm}^{-1}$; HMRS (ESI) calcd for $\mathrm{C}_{17} \mathrm{H}_{21} \mathrm{~N}_{2} \mathrm{O}_{2}[\mathrm{M}+\mathrm{H}]^{+}$: 285.1595. Found: 285.1597.<smiles>O=C(NO)Nc1ccc(C(=O)O)cc1</smiles>

1-Hydroxy-3-(4-nitrophenyl)urea (38). The title compound was prepared from 4-nitrobenzoic acid (167 $\mathrm{mg}, 1.00 \mathrm{mmol}$ ) and $\mathrm{N}$-Hydroxylamine hydrochloride (139 $\mathrm{mg}, 1.00 \mathrm{mmol}$ ) according to the general procedure. After 20 hours of reaction, a $10 \%$ solution of $\mathrm{NaNO}_{2}(20 \mathrm{ml})$ was added. The mixture was then diluted with ethyl acetate $(20 \mathrm{ml})$, and the resulting mixture was stirred during 20 min at $25^{\circ} \mathrm{C}$. The aqueous layer was extracted with ethyl acetate $(3 \times 30 \mathrm{ml})$ The combined organic layers were washed with saturated $\mathrm{NH}_{4} \mathrm{Cl}(2 \times 20 \mathrm{ml})$, and brine $(20 \mathrm{~mL})$. The organic layer was dried over $\mathrm{Na}_{2} \mathrm{SO}_{4}$ and the solvent was removed under reduced pressure to afford a orange oil. The desired urea 38 (105 mg, 55\%) was obtained as a yellow solid, after flash chromatography $\left(5 \% \mathrm{MeOH} / \mathrm{CHCl}_{3}\right) . \mathrm{R}_{f} 0.51$ $\left(5 \% \mathrm{MeOH} / \mathrm{CHCl}_{3}\right.$ ); mp $140{ }^{\circ} \mathrm{C} ;{ }^{1} \mathrm{H} \mathrm{NMR}$ (400 MHz, DMSO) $\delta 9.50$ (s, 1H), 9.28 (s, 1H), 9.15 (s, $1 \mathrm{H}), 8.15(\mathrm{~d}, J=9 \mathrm{~Hz}, 2 \mathrm{H}), 7.92(\mathrm{~d}, J=9 \mathrm{~Hz}, 2 \mathrm{H}) ;{ }^{13} \mathrm{C}$ NMR $(100 \mathrm{MHz}, \mathrm{DMSO}) \delta 157.6,146.3$, 141.1, 124.7, 118.3; IR 3288, 2938, 2835, 1628, 1508, 1443, 1245, 1167, 1026, 842, $768 \mathrm{~cm}^{-1}$; HMR (ESI) calcd for $\mathrm{C}_{7} \mathrm{H}_{8} \mathrm{~N}_{3} \mathrm{O}_{4}[\mathrm{M}+\mathrm{H}]^{+}$: 198.0509. Found: 198.0512 .
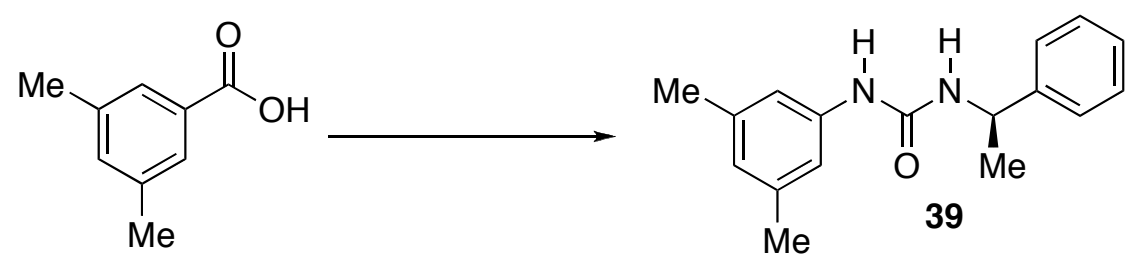

1-(3,5-Dimethylphenyl)-3-((R)-1-phenylethyl)urea (39). The title compound was prepared from 3,5-dimethylbenzoic acid $(150 \mathrm{mg}, 1.00 \mathrm{mmol})$ and $(R)$-methylbenzyl amine $(191 \mu \mathrm{L}, 1.50 \mathrm{mmol})$ according to the general procedure. The desired urea $39(182 \mathrm{mg}, 63 \%)$ was obtained as a white solid after filtration. $\mathrm{R}_{f} 0.69(40 \% \mathrm{EtOAc} /$ hexanes $) ; \mathrm{mp} 167^{\circ} \mathrm{C} ;[\alpha]_{\mathrm{D}}{ }^{25}=+6.6(\mathrm{c} 1.0, \mathrm{MeOH}) ;{ }^{1} \mathrm{H} \mathrm{NMR}(400$ MHz, DMSO) $\delta 8.22(\mathrm{~s}, 1 \mathrm{H}), 7.33-7.32(\mathrm{~m}, 4 \mathrm{H}), 7.25-7.21(\mathrm{~m}, 1 \mathrm{H}), 6.97(\mathrm{~s}, 2 \mathrm{H}), 6.58(\mathrm{~d}, J=8 \mathrm{~Hz}$, $1 \mathrm{H}), 6.52(\mathrm{~s}, 1 \mathrm{H}), 4.80-4.76(\mathrm{~m}, 1 \mathrm{H}), 2.17(\mathrm{~s}, 6 \mathrm{H}), 1.37(\mathrm{~d}, J=7 \mathrm{~Hz}, 3 \mathrm{H}) ;{ }^{13} \mathrm{C} \mathrm{NMR}(100 \mathrm{MHz}$, DMSO) $\delta 154.3,145,2,140.2,137.4,128.2,126.5,125.7,122.6,115.2,48.5,23.0,21.0$; IR 3305, 2968, 2922, 1632, 1558, 1445, 1231, 850, $685 \mathrm{~cm}^{-1}$; HMRS (ESI) calcd for $\mathrm{C}_{17} \mathrm{H}_{20} \mathrm{~N}_{2} \mathrm{O}[\mathrm{M}+\mathrm{H}]^{+}$: 269.1652. Found: 269.1652. 